Polymer Journal, Vol. 5, No. 3, pp 283-287 (1973)

\title{
Stress Relaxation of Polymer Solutions under Large Strain: Application of Double-Step Strain*
}

\author{
Kunihiro Osaki, Yoshiyuki Einaga, ${ }^{* *}$ and Michio Kurata \\ Institute for Chemical Research, Kyoto University, \\ Uji, Kyoto 611, Japan. \\ Nobuhiro YAMADA*** and Mikio TAMURA \\ Department of Industrial Chemistry, Kyoto University, \\ Sakyo-ku, Kyoto 606, Japan.
}

(Received May 10, 1973)

\begin{abstract}
Stress relaxation was measured after application of a double-step shear strain on a concentrated solution of polystyrene in chlorinated biphenyl. The first shear strain $s_{1}$ was applied to the sample in a cone-plate sample holder of a relaxometer at time $-t_{1}$, the second strain $s_{2}$ was added at $t=0$, and then the stress was measured as a function of time $t$. Ranges of $s_{1}$ and $t_{1}$ were 1.71-20.5 shear units and 10-5000 sec, respectively, while $s_{2}$ was kept constant at 1.88 s.u. These results, together with published results for strain-dependent relaxation moduli, were employed to assess a group of constitutive equations which use single integrals with respect to the time $t^{\prime}$ to describe the strain history. It was shown that such constitutive equation could not in general describe the observed results, when invariants at $t^{\prime}$ of the strain rate or the strain were employed in the response functions to represent the nonlinear behavior. However, in the special cases of relatively small $s_{1}$ or large $t_{1}$, constitutive equations of the single integral type described quantitatively the observed nonlinear behavior, when invariants of strain were employed in the response functions.
\end{abstract}

KEY WORDS Stress Relaxation / Constitutive Equation / Nonlinear Behavior / Double-Step Strain / Polystyrene / Solution !

A variety of constitutive equations in differential as well as integral forms have been proposed to describe the nonlinear viscoelastic behavior of polymeric systems. ${ }^{1}$ Among the equations, those of the single integral type are of special importance, due to their simplicity and also to their generality in including the phenomenological theory of linear viscoelasticity. Equations of this type may be written as

$$
\boldsymbol{\sigma}(t)=\int_{-\infty}^{t} \sum_{i} R_{i}\left(t-t^{\prime} ; A\right) \mathbf{S}_{i}\left(t^{\prime}\right) \mathrm{d} t^{\prime}
$$

where $\boldsymbol{\sigma}(t)$ is the stress tensor at time $t, \mathbf{S}_{i}\left(t^{\prime}\right)$ is a tensor derived from the strain tensor at $t^{\prime}$

\footnotetext{
* Requests for reprints should be addressed to $\mathbf{M}$. Kurata.

** Present address: Department of Polymer Science, Osaka University, Toyonaka, Osaka.

*** Present address: Nippon Tokushu Kento Ltd., Kisshoin, Minami, Kyoto.
}

defined relative to the state at $t, R_{i}$ is a response function representing the properties of the material and $\Sigma_{i}$ indicates that the integrand may be composed of several terms with different tensors $\mathbf{S}_{i}\left(t^{\prime}\right)$. The quantity $A$ represents the magnitude of strain or strain rate and is a scalar derived from the invariants of the strain or strain-rate tensor. Published equations of this type may be classified into three categories according to the definition of $A:^{2}$ (1) $A$ is a combination of the invariants of the strain-rate tensor at $t^{\prime}$, (2) $A$ is a combination of an average of the invariants of the strain-rate tensor between $t^{\prime}$ and $t$, and (3) $A$ is a combination of the invariants of the strain tensor at $t^{\prime}$ defined relative to the state at $t$. Classes (1) and (3) probably represent the simplest equations of the single integral týpe which are able to describe any non-linear behavior, because in these 
cases response functions are defined only in reference to two states $t^{\prime}$ and $t$.

Yamamoto recently examined the applicability of equations of class (1) to polymer solutions and pointed out the importance of obtaining rate-dependent relaxation spectra. ${ }^{2,3}$ However, recent experimental results on stress relaxation under large strain have revealed that equations of class (1) may not be applicable to deformations of a discontinuous nature without large and intricate modifications. ${ }^{4,5}$ It has also been shown that the strain-dependent relaxation spectrum of Yamamoto ${ }^{6}$ is easily obtained from the strain-dependent relaxation modulus, if an equation of class (3) is applied. ${ }^{5}$ However, it remains uncertain whether the constitutive equation of class (3) thus obtained is suitable for describing the nonlinear behavior of polymeric systems with a different strain history. In order to test the applicability of an equation of class (3), we measured, in the present study, the stress relaxation after application of a two-step strain and studied the effect of strain history, such as the magnitude of the first-step strain and the time interval between two steps. The stress relaxation after application of a two-step strain has been reported by Zapas and Craft for elongational deformation of polyisobutylene and plasticized poly(vinyl chloride). ${ }^{7}$ They observed that the BKZ theory, ${ }^{8}$ a special case of class (3), is applicable to these systems. Our experimental results extended up to larger values of the strain than did those of Zapas and Craft.

\section{EXPERIMENTAL}

The stress relaxation was measured after application of a two-step strain in a solution of polystyrene in Aroclor 1248. The polystyrene was the standard sample $14 \mathrm{a}$, of molecular weight $1.80 \times 10^{6}$, supplied by Pressure Chemical Company. The solvent, Aroclor 1248, was a mixture of partially chlorinated biphenyl supplied by Monsanto Chemical Company. The solution was the same as that previously used for measurements of strain-dependent relaxation moduli. ${ }^{4,5}$ Experiments were performed at $30^{\circ}$. Measurements were carried out with a coneplate type relaxometer as reported earlier. ${ }^{9}$ The double-step strain was applied by repeating

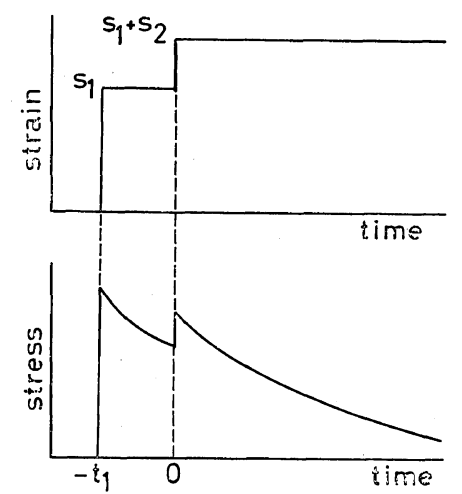

Figure 1. Illustration of stress relaxation for double-step strain. The first strain $s_{1}$ is applied at time $t=-t_{1}$ and the second strain $s_{2}$ is added at $t=0$. Stress is measured as a function of time for $t>0$.

twice the procedure for the usual (single-step) relaxation measurement. The first strain $s_{1}$ was applied at time $t=-t_{1}$, the second strain $s_{2}$ was added at $t=0$ and then the shear stress $\sigma$ was measured as a function of time, as illustrated in Figure 1. The second strain $s_{2}$ was kept small ( $s_{2}=1.71$ or 1.88 shear units) in the present measurements, while the first strain $s_{1}$ and the time interval $t_{1}$ were varied from 1.71 to 20.5 s.u. and from 10 to $5000 \mathrm{sec}$.

\section{RESULTS}

Examples of the stress relaxation for the double-step strain are shown in Figures 2 through 4 for various combinations of the first strain $s_{1}$ and the time interval $t_{1}$. Figure 2 gives the results for $s_{1}=1.71$ and $s_{2}=1.71$. Different symbols represent various values of $t_{1}$. The stress $\sigma$ for $t_{1}=0$ (filled circles) is the same as that for a single-step strain of magnitude $s=$ $s_{1}+s_{2}$. On the other hand, $\sigma$ for $t_{1}=5000 \mathrm{sec}$ (circles with pip up) is equal within experimental error to that for a single-step strain of $s=s_{2}$. This result is consistent with the previous result ${ }^{5}$ that the maximum relaxation time for this system, approximately $1.5 \times 10^{3} \mathrm{sec}$, is much shorter than $5000 \mathrm{sec}$. If the relation of linear viscoelasticity applies, the stress for $t_{1}=0 \mathrm{sec}$ should be larger than that for $t_{1}=5000 \mathrm{sec}$ by a factor 2 over the whole range of times, and the stress for intermediate $t_{1}$ should be intermediate to those for $t_{1}=0$ and $5000 \mathrm{sec}$. How- 


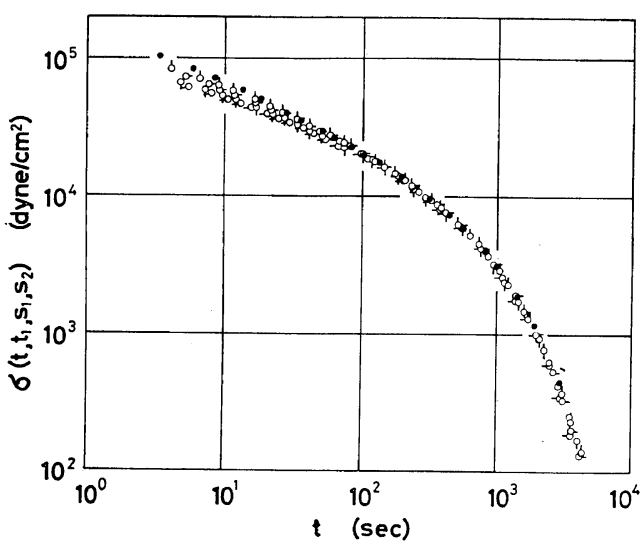

Figure 2. Stress for double-step strain measured for $20 \%$ solution of polystyrene $14 \mathrm{a}$ at $30^{\circ}$. The first strain $s_{1}$ and the second strain $s_{2}$ are both 1.71 shear unit. Various directions of pips represent time intervals $t_{1}$ : pip up, $5000 \mathrm{sec}$; successive $90^{\circ}$ rotations clockwise correspond to 1000, 100, and $20 \mathrm{sec}$, respectively. Filled circles represent $t_{1}=0$.

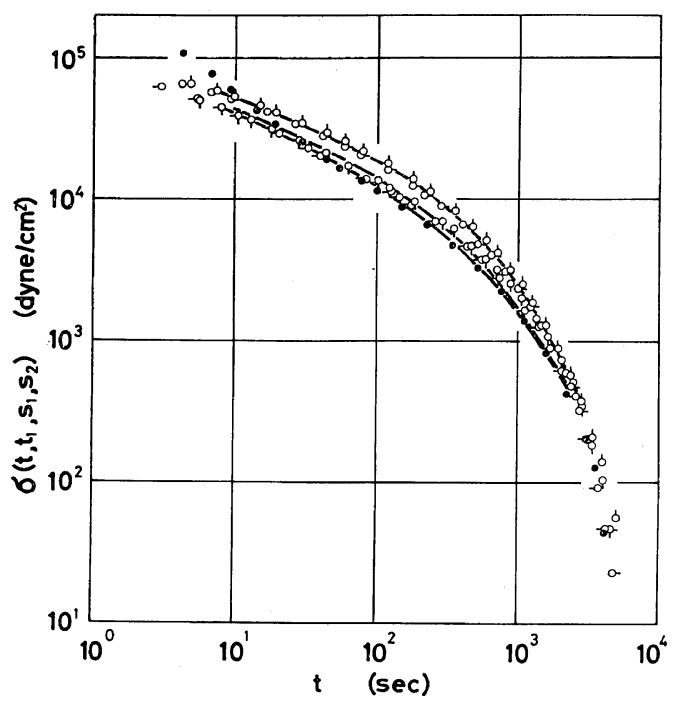

Figure 3. Stress for double-step strain, measured for $20 \%$ solution of polystyrene $14 \mathrm{a}$ at $30^{\circ}$. The first strain $s_{1}$ is 6.83 and the second strain $s_{2}$ is 1.88 shear units. Various directions of pips represent time intervals $t_{1}$ : pip up, $5000 \mathrm{sec}$; successive $90^{\circ}$ rotations clockwise correspond to 1000 , 100 , and $20 \mathrm{sec}$, respectively. Filled circles represent $t_{1}=0$. Solid lines represent predictions of eq 5 for $t_{1}=1000,100$, and $20 \mathrm{sec}$, from top to bottom.

Polymer J., Vol. 5, No. 3, 1973

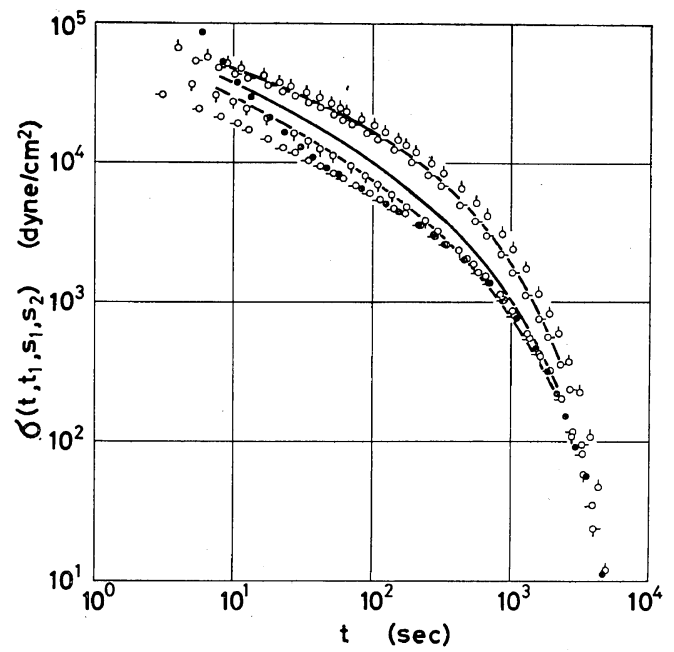

Figure 4. Stress for double-step strain, measured for $20 \%$ solution of polystyrene $14 a$ at $30^{\circ}$. The first strain $s_{1}$ is 20.5 and the second strain $s_{2}$ is 1.88 shear units. Various directions of pips represent time intervals $t_{1}$ : pip up, $5000 \mathrm{sec}$; successive $90^{\circ}$ rotations clockwise correspond to 1000 , 100 , and $20 \mathrm{sec}$, respectively. Filled circles represent $t_{1}=0$. Solid lines represent predictions of eq 5 for $t_{1}=1000,100$, and $20 \mathrm{sec}$, from top to bottom.

ever, the observed stress for $t_{1}=0$ is larger than that for $t_{1}=5000 \mathrm{sec}$ only in the range of very short times. In the range of longer times, these two stresses are approximately equal to each other. This result might be expected from the fact that the strain-dependent relaxation modulus $G(t, s)$ is relatively insensitive to the variation of the strain $s$ when $t$ is small but decreases in proportion to $\left(s^{2}+3\right)^{-0.83}$ when $s$ and $t$ are large. ${ }^{4,5}$ Therefore, the stress $s G(t, s)$ for a single-step strain at long times increases as $s$ increases from 0 to about 2 and then decreases as $s$ increases. In the case of Figure 2, the stresses for a single-step strain at long times are insensitive to the variation of $s$ because $s$ is close to 2 . The stress for the double-step strain of intermediate time interval $t_{1}$ is approximately equal to that for the single-step strain (or $t_{1}=$ $5000 \mathrm{sec})$ in the range of long times $\left(t>10^{2} \mathrm{sec}\right)$. In other words, the strain history preceding the application of $s_{2}$ does not affect the stresses in this range of long times. In the range of shorter times, however, $\sigma$ decreases as $t_{1}$ decreases; the 
smaller the observed stress, the larger the stress remaining at the time of application of the additional strain.

Figure 3 shows the results for the combination of $s_{1}=6.83$ and $s_{2}=1.88$. In contrast to the case in Figure 2, the stress for $t_{1}=0 \mathrm{sec}$ is smaller than those for other values of $t_{1}$ in the range of long times. This is expected, because $s$ exceeds 2 in this case. Again, the larger the stress remaining at the time of application of the second strain, the larger is the observed stress on the short time scale. In this case, the effect of the variation of $t_{1}$ persists to a longer time scale than was the case in Figure 2. Figure 4 represents the result for a still larger $s_{1}: s_{1}=$ 20.5 and $s_{2}=1.88$. Qualitatively the same features as in Figure 3 are observed. However, it is clear that the effect of the variation of $t_{1}$ is larger and persists to still longer times than in Figure 3.

\section{DISCUSSIONS}

In order to examine the applicability of a constitutive equation of class (3), as defined in the introductory section, let us rewrite eq 1 for the shear stress in a simple shear deformation. Since functions $A$ and $\mathbf{S}_{i}$ for a simple shear deformation include only the shear strain $s\left(t^{\prime}\right)$ as a variable in the case of class (3), $\sigma$ may be written as

$$
\sigma(t)=\int_{-\infty}^{t} F\left(t-t^{\prime} ; s\left(t^{\prime}\right)\right) \mathrm{d} t^{\prime}
$$

where $s\left(t^{\prime}\right)$ is the strain at $t^{\prime}$ defined relative to the state at $t$ and $F$ is a scalar function of $t-$ $t^{\prime}$ and $s\left(t^{\prime}\right)$. By introducing the strain defined in Figure 1 for $s\left(t^{\prime}\right)$, one obtains an expression for the stress for a double-step strain as

$$
\sigma(t)=\sigma\left(t+t_{1}, s_{1}+s_{2}\right)-\sigma\left(t+t_{1}, s_{2}\right)+\sigma\left(t, s_{2}\right)
$$

where $\sigma(t, s)$ is the stress at time $t$ after application of a single-step strain $s$. The stress $\sigma(t, s)$ is related to the strain-dependent relaxation modulus $G(t, s)$ as $^{4}$

$$
\sigma(t, s)=s G(t, s)
$$

In the case of linear viscoelasticity, $G(t, s)$ is independent of $s$ and eq 3 reduces to

$$
\sigma(t)=s_{1} G\left(t+t_{1}\right)+s_{2} G(t)
$$

where $G(t)$ is the relaxation modulus of linear viscoelasticity.

With the use of $G(t, s)$ reported earlier, the stresses for a double-step strain were calculated with eq 3 and were compared with the experimental results. Examples of calculated results are shown by the solid lines in Figures 3 and 4. In the case of small $s_{1}$ and $s_{2}$, the agreement between theoretical and experimental results is excellent, irrespective of the time interval $t_{1}$, although calculated results are not shown in Figure 2. The agreement is also good for moderately large values of $s_{1}$, as shown in Figure 3. It might be remarked that eq 5 for linear viscoelasticity gives results in the wrong direction: the theoretical value of $\sigma(t)$ decreases as $t_{1}$ increases. When the strain $s_{1}$ is large, eq 3 shows a tendency to overestimate the stress for a double-step strain in the range of short times, as shown in Figure 4. The relative discrepancy between theoretical and observed results is larger when the time interval $t_{1}$ is smaller. For $t_{1}=1000 \mathrm{sec}$, predicted values are in close agreement with those observed. This agreement may be partly trivial, because the stress should approach $\sigma\left(t, s_{2}\right)$ as $t_{1}$ increases, for constitutive equations of any type. Still, the contribution of the first two terms. on the right hand side of eq 3 is essential for the agreement, as seen by the fact that the calculated result is clearly closer to the observed than is $\sigma\left(t, s_{2}\right)$ represented by the result for $t_{1}=5000 \mathrm{sec}$.

It may be concluded that no constitutive equation of class (3) is capable of describing stresses at very large deformations of the typical polymer solution studied in this study. The success of the BKZ model in describing stress relaxation for a double-step strain is apparently due to the fact that the measurements were confined to relatively small strains. ${ }^{7}$ As mentioned before, classes (1) and (3) represent the simplest equations of the single integral type that involve response functions defined in reference to only two states $t^{\prime}$ and $t$. On the other hand, eq 3 may be applied to any equations of the single integral type with response functions defined in reference to two states $t^{\prime}$ and $t$, although the derivation is not shown 
here. Therefore, the present result indicates that response functions for the polymer solution should require as variables at least two states of the strain history, in addition to the state $t$. In other words, each value of the response function may be determined by giving strain states of at least two times in addition to the state at time $t$ of observation of stress. When the strain is not too large, an equation of class (3) is capable of describing the shear stress in a double-step shear strain. It is possible that the constitutive equation of class (3) is a good approximation for describing any stress component for any strain history in polymer solutions in a restricted range of magnitude of the strain. We will examine this possibility in subsequent publications making use of various strain histories.

\section{REFERENCES}

1. See for example, A. S. Lodge, "Elastic Liquids," Academic Press, London, 1964; T. W. Spriggs, J. D. Huppler, and R.B. Bird, Trans. Soc. Rheology, 10, 191 (1966); D. C. Bogue and J. Doughty, Ind. Eng. Chem. Fund., 5, 243 (1966); M. Yamamoto, "Buttai no Henkeigaku," Seibundo-Shinkosha, Tokyo, 1972.

2. M. Yamamoto, Trans. Soc. Rheology, 15, 331 (1971).

3. M. Yamamoto, ibid., 15, 783 (1971).

4. Y. Einaga, K. Osaki, M. Kurata, S. Kimura, and M. Tamura, Polymer J., 2, 550 (1971).

5. Y. Einaga, K. Osaki, M. Kurata, S. Kimura, N. Yamada, and M. Tamura, ibid., 5, 91 (1973).

6. M. Yamamoto, Zairyo, 21, 355 (1972).

7. L. J. Zapas and T. Craft, J. Res. Nat. Bur. Stand., A, 69, 541 (1965).

8. B. Bernstein, E. A. Kearsley, and L. J. Zapas, Trans. Soc. Rheology, 7, 391 (1963).

9. M. Tamura, M. Kurata, K. Osaki, Y. Einaga, and S. Kimura, Bull. Inst. Chem. Res. Kyoto Univ., 49, 43 (1971). 